\title{
Dogs Can Be Successfully Trained to Alert to Hypoglycemia Samples from Patients with Type 1 Diabetes
}

Dana S. Hardin $\cdot$ Wesley Anderson $\cdot$ Jennifer Cattet

To view enhanced content go to www.diabetestherapy-open.com

Received: July 21, 2015 / Published online: October 6, 2015

(C) The Author(s) 2015. This article is published with open access at Springerlink.com

\section{ABSTRACT}

Introduction: Hypoglycemia (Hypo) is the most common side effect of insulin therapy in people with type 1 diabetes (T1D). Over time, patients with T1D become unaware of signs and symptoms of Hypo. Hypo unawareness leads to morbidity and mortality. Diabetes alert dogs (DADs) represent a unique way to help patients with Hypo unawareness. Our group has previously presented data in abstract form which demonstrates the sensitivity and specificity of DADS. The purpose of our current study is to expand evaluation of DAD sensitivity and specificity using a method that reduces the possibility of trainer bias.

Methods: We evaluated 6 dogs aging 1-10 years old who had received an average of 6 months of training for Hypo alert using positive training

Electronic supplementary material The online version of this article (doi:10.1007/s13300-015-0135-x) contains supplementary material, which is available to authorized users.

D. S. Hardin $(\varangle)$. W. Anderson

Eli Lilly, Corp 225 S. Delaware, Indianapolis, USA

e-mail: hardin_dana_sue@lilly.com

J. Cattet

Medical Mutts, Indianapolis, Indiana, USA methods. Perspiration samples were collected from patients during Hypo (BG 46-65 mg/dL) and normoglycemia (BG $85-136 \mathrm{mg} / \mathrm{dl}$ ) and were used in training. These samples were placed in glass vials which were then placed into 7 steel cans (1 Hypo, 2 normal, 4 blank) randomly placed by roll of a dice. The dogs alerted by either sitting in front of, or pushing, the can containing the Hypo sample. Dogs were rewarded for appropriate recognition of the Hypo samples using a food treat via a remote control dispenser. The results were videotaped and statistically evaluated for sensitivity (proportion of lows correctly alerted, "true positive rate") and specificity (proportion of blanks + normal samples not alerted, "true negative rate") calculated after pooling data across all trials for all dogs.

Results: All DADs displayed statistically significant ( $p$ value $<0.05$ ) greater sensitivity (min 50.0\%-max 87.5\%) to detect the Hypo sample than the expected random correct alert of $14 \%$. Specificity ranged from a min of $89.6 \%$ to a max of $97.9 \%$ (expected rate is not defined in this scenario).

Conclusions: Our results suggest that properly trained DADs can successfully recognize and 
alert to Hypo in an in vitro setting using smell alone.

Keywords: DADS diabetes; Diabetes alert dogs; Dogs; Hypoglycemia; Service dogs; Type 1 diabetes

\section{INTRODUCTION}

Hypoglycemia (Hypo) is the most common side effect of insulin therapy, particularly with intensive insulin management which is the recommended regimen for patients with type 1 diabetes (T1D) [1]. Episodes are often distressing and carry the risk of serious neurological and cardiovascular sequelae [1]. Hypo is especially dangerous in patients who have lost the early warning symptoms of falling blood glucose levels (Hypo unawareness) [1]. Episodes of Hypo occurring at night have been cited as a cause of death [2]; Hypo is one of the most feared complications of diabetes. Fear of Hypo can result in patients not taking adequate insulin [3]. Ever-expanding technology, including more accurate glucometers and continuous glucose monitors $[4,5]$, has helped patients proactively monitor for Hypo, yet there is always a need for additional methods for use in different situations to help patients prevent serious Hypo,

Dogs represent a unique alerting system for Hypo. One published report indicates that over one-third of dogs living with a diabetic, even dogs not specifically trained for the task, demonstrate behavioral changes during their owner's Hypo episodes [6]. Dogs which are specifically trained to alert for Hypo are called diabetes alert dogs (DADs). There has been multiple anecdotal or case reports [7-11] describing the benefits of these trained dogs as an alerting system. Our group previously presented an abstract [7] which described the clinical impact of DADs by presenting results of a proprietary questionnaire given to patients before and after DAD placement. Despite these reports, it is clear that more robust demonstration of success is needed before DADs can be validated as a potential tool to aid patients with diabetes. The purpose of our current study was to test the hypothesis that dogs can be successfully trained to alert to Hypo samples collected from people with T1D.

\section{METHODS}

\section{Selection of Dogs}

Our group utilizes dogs from shelters to train as DADs and all of them were trained for obedience for later certification for public access. Dogs which were trained for this study were selected by the Medical Mutts Director and Trainer (JC) based on personality traits identified as compatible for service dog training $[12,13]$. Some of these traits included sociability, trainability, adaptability and confidence. The dogs were also selected based on their physical aptitude for the job, such as size, health and shape of their noses. Selected dogs were 9-18 months of age to assure sufficient maturity for assessing temperament.

\section{Collection of Patient Samples Used for Training}

Samples were collected from 4 patients with T1D and were used for up to 2 weeks for ongoing training. Patients were instructed on collection of samples by personal instruction with endocrinologist (DSH). They were also provided a written instruction sheet describing sample collection, as well as a YouTube link 
reviewing the procedure. Patients were instructed to use a gauze pad $(2 \times 2$, Johnson and Johnson, New Brunswick, NJ, USA) to wipe their forehead and back of the neck, then to place the pad into a zip-locked bag (Ziplock brand, SC Johnson, Racine, WI, USA). Immediately after placing the sample into the bag, the patient also blew an exhaled breath into the bag before sealing it. Each bag contained a label on which patients wrote their initials, date/time of collection and their corresponding blood sugar value. Patients were instructed to deliver samples to a central site within in $48 \mathrm{~h}$ of collection. The samples were stored in the freezer (Temperature $0{ }^{\circ} \mathrm{F},-18^{\circ} \mathrm{C}$ ) until use.

Patients were instructed to collect samples during Hypo and normoglycemia. The reference ranges for Hypo- and normo-glycemia were given according to American Diabetes Association guidelines [14] (Hypo: $<70 \mathrm{mg} / \mathrm{dl}$; normo: fasting $<100 \mathrm{mg} / \mathrm{dl}$; pre-prandial $80-130 \mathrm{mg} / \mathrm{dl}$; post-prandial $<180 \mathrm{mg} / \mathrm{dl}$ ). Patients measured their blood glucose levels using their home glucometers and were trained on the use and quality control of their glucometers by their health care professionals. Patients were instructed to collect the Hypo samples only if they felt able to do so and to collect the sample within $5 \mathrm{~min}$ of the occurrence, but only after confirmation of the hypoglycemic event as measured by their glucometer. Once the Hypo sample was collected, patients were instructed to wait a minimum of $8 \mathrm{~h}$ before collecting a sample for normoglycemia. They were also instructed not to wait longer than $24 \mathrm{~h}$ to collect the normoglycemic samples. Samples used in our study were collected according to an Institutional Review Board approved protocol.

\section{Training}

The DADs used for this study were trained using positive reinforcement methods $[12,13]$, using a clicker (marker) paired with a reward (food treat). Coercion-based methods were avoided so as not to increase the chance of the dog developing anxiety, fears, or resistance to certain situations or to certain tasks [15]. Dogs were equipped with flat quick release collars and a front clip harness.

The dogs were trained for good behavior according to established methods [16]. This training included how not to be bothered by sudden noises, children running by, or by people trying to interact with them. They were taught to lie quietly under the table when at the restaurant or at the office, to walk within a foot of the handler without pulling on the leash and to ignore people or other animals. During their training, they learned 30 commands, including sit, down, stay, under (to lie down under the table), hurry (to potty on cue in designated areas), come, stand, touch, bed (go to the dog's sleeping place), heel, side, jump, off, crawl, find help, get/give (retrieval of different items such as a phone, glucose tablets or a small bag), etc.

Specific training for Hypo alert was done in three phases. The first phase was designed to teach the dog to recognize the Hypo samples by introducing them to the skin and breath samples collected from patients (as described above). During this initial training, the samples were placed in a glass vial. The dog was taught to put its nose on the vial and immediately alert by sitting. If the dog alerted correctly to the Hypo sample, it was rewarded with a food treat (positive reinforcement). Once the behavior of touching and sitting was automatic, in other words, no longer required prompting from the trainer, the second phase of training was begun. 
In the second phase, the vial containing the Hypo sample was placed in a one gallon-sized steel can. The dog was expected to go to the can, followed by smelling the vial then sitting. Once the dog sat appropriately in front of the can containing the Hypo sample, a food reward was given. Once this task worked repeatedly for the single sample, additional cans containing identical vials were gradually introduced. Each of these additional cans and vials contained either blank or normoglycemia samples. The additional samples were from the same person as the Hypo sample. The introduction of each additional sample was dependent on the improvement of the dog's ability to understand the discrimination task (i.e., repeatedly alert to the Hypo sample, but not to the other sample(s).

In the third phase of training, the glass vials containing samples were placed on a person. The sitting alert was replaced by teaching the dog to alert by poke of the nose on the person's body. (Specific to this study: some of the dogs participating in this study were already in their final stages of training, and were conditioned to poke a person when detecting Hypo. Thus, it was decided that said dogs would use their nose to push the can to indicate which can contained the Hypo sample, instead of sitting, to avoid potential confusion and set-backs in their training). During all training, the appropriate alert was rewarded by the trainer using a food treat and praise.

Once the dog was trained competently to alert to Hypo at all three phases of the training; the dog was introduced to samples from additional patients and the process of training continued until the dog was competently able to distinguish Hypo samples from normoglycemia samples from multiple patients. Once the dog was fully trained to recognize Hypo from multiple patients, able to localize to the body and provide the trained alert, the dog was then called a DAD and at that point could be considered for placement with a diabetic person. For our study, we used fully trained DADs.

\section{Testing Environment and Protocol}

We created a specific testing environment for purposes of this study (Fig. 1). During testing, the dogs were placed in a closed room, separate from any human, which contained the samples as described below. The dogs were rewarded for successful identification of the Hypo sample with a treat from an automatic dispenser remotely activated by the trainer: the Pet Tutor $^{\circledR}$ (Indianapolis, IN, USA). This measure removed the need for a person in the test room, thus also decreasing possible sources of distraction, or information, to the dog.

The cans in the room were arranged as follows. One Hypo sample was placed in one steel can, normoglycemia samples were placed in two cans, and four cans contained a blank gauze pad. The cans were placed on the floor in a semi-circle one foot apart. The order of placement was determined by roll of the dice. The semi-circle of cans was placed facing the entrance of the room 15 feet from room entry. The Pet Tutor was set on the floor, facing the cans, at an approximate distance of 8 feet. The dogs were led into the test room, on their own, and the door was closed behind them. Monitoring of their behavior was done by the experimenter with the use of a video camera and reinforcement of the accurate alert was provided by remote dispense of a treat from the Pet Tutor. The dogs were called out of the test room soon after being rewarded. The results were videotaped using a video camera placed in the closed room.

Dogs were tested in random fashion and testing occurred for a maximum of 1 min per 


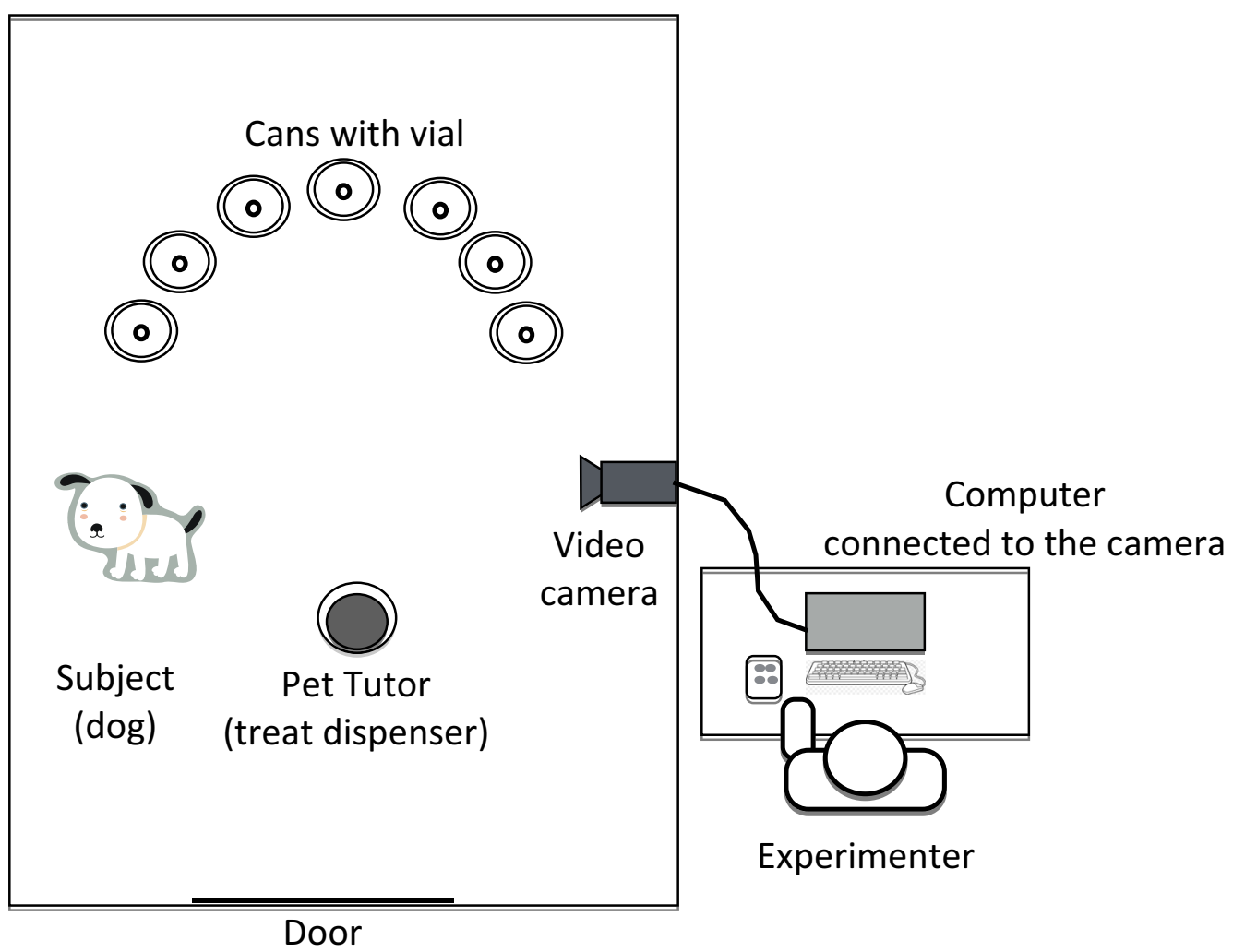

Fig. 1 Room layout for testing DADs

trial. Each dog was tested eight times (4 patient samples $\times 2$ replicated trials). Dogs were given a 2-h break after the first four tests.

\section{Statistical evaluation of this study}

The videotaped recordings of the dog's detection of the Hypo sample were reviewed and scored by the statistician (W.A.). Sensitivity (proportion of lows correctly alerted, "True positive rate") and specificity (proportion of non-low samples: blanks or normal samples not alerted, "True negative rate") calculated after pooling data across all trials for all dogs.

Specificity was determined as the proportion of non-low samples (blanks or normal samples not alerted, the "true negative" rate).

Each trial began with randomized placement of samples in each of the cans. For the second trial (a replicate), random placement again occurred. Scoring rules for trial outcomes can be found in Table 1. Statistical results were calculated using JMP 12 software (SAS Inc., Carey, NC, USA) and reported similarly to results published from cancer-detecting dogs [17].

\section{RESULTS}

For this analysis, six dogs were evaluated and each dog was studied eight times. Table 2 presents the breed/mix of dogs, gender and age.

One of seven sample cups (14.29\%) contained the Hypo sample. Therefore, the expected random chance alert sensitivity level was $14 \%$. All DADS displayed a statistically significantly greater sensitivity (50.0\%-87.5\%) to detect the Hypo sample than the expected random correct alert. 
Table 1 Scoring rules for DAD actions within a trial

\begin{tabular}{lllr}
\hline Scenario & Action by DAD & Low sample & $\begin{array}{c}\text { Non-low sample } \\
\text { (blank, normal) }\end{array}$ \\
\hline 1 & 1 st alert & True positive (TP) & False positive (FP) \\
2 & Subsequent alerts ${ }^{\mathrm{a}}$ & False negative (FN) (since not 1st alert) & False positive (FP) \\
3 & No alert (within time) & False negative (FN) & True negative (TN) \\
4 & No alert timeout (no action by DAD) & False negative (FN) & True negative (TN) \\
\hline
\end{tabular}

In our recent study (\#3) there were no scenarios for \#4, i.e., all dogs gave an alert before the end of time (60 s)

$D A D s$ diabetes alert dogs

a For "subsequent alerts" within the same trial this scoring is conservative and may be thought of as a penalty for guessing. For example, if the dog did alert on the low but only after alerting on a non-low the result is scored as miss or false negative. 12 of 60 alerts or $20 \%$ were subsequent alerts and varied by dog

Sensitivity $=100 \% \times \frac{\text { True Positives }}{\text { True Positives }+ \text { False Negatives }}$ Specificity $=100 \% \times \frac{\text { True Negatives }}{\text { True Negatives }+ \text { False Positives }}$

Table 2 BREED/MIX of DADs used in the representative results trial

\begin{tabular}{lcl}
\hline Breed of dog & Age & Gender \\
\hline Labrador retriever & 2 & Male \\
Flat coated retriever & 2 & Female \\
Siberian husky mix & 1.5 & Female \\
Spaniel mix & 1 & Male \\
German shepherd & 10 & Female \\
Labrador retriever & 2 & female \\
\hline
\end{tabular}

$D A D s$ diabetes alert dogs

Specificity ranged from $50.0 \%$ to $89.6 \%$. Overall, the best four dogs performed at $87.5 \%$ sensitivity and $97.9 \%$ specificity. The dog with the poorest performance displayed $50.0 \%$ sensitivity and $89.6 \%$ specificity. Results are presented in Table 3.

\section{DISCUSSION}

Our results demonstrate that DADs are able to identify Hypo and be trained to alert to its presence. The results reported here take canine glucose sensing to a new level of sophistication. Using the Pet Tutor, a remote-controlled treat-dispensing device which removed human interaction during the testing, we have clearly been able to demonstrate that DADs sense Hypo accurately due to smell alone. Once DADs are placed with their owner/patient, it also likely DADs will cue to certain behavioral clues exhibited by the patient and these cues would also likely serve to help the DAD properly alert the owner of impending Hypo.

The results from our current trial are better than those we presented in a previous abstract [7]. Potential reasons for the better performance by these DADs include: (1) One dog participating in the trial presented in our prior abstract was new to training, and young. Her poor performance had a lot of influence on the overall results from our prior trial of four dogs, (2) in our current study, we used containers with materials which do not collect previous scent and never re-used a Hypo container for any other scent (blank or normal), (3) instead of using a Lazy Susan device, which provides less area to spread out the containers, we used random placement where the containers were more spaced out, thus minimizing potential overlap of the scent plume, (4) in the current 
Table 3 Sensitivity and specificity for Six DADs evaluated for representative results

\begin{tabular}{llclllllll}
\hline Dog & Total samples & $\begin{array}{l}\mathbf{N} \\
(\mathbf{F N})\end{array}$ & $\begin{array}{l}\mathbf{N} \\
(\mathbf{F P})\end{array}$ & $\begin{array}{l}\mathbf{N} \\
(\mathbf{T N})\end{array}$ & $\begin{array}{l}\mathbf{N} \\
(\mathbf{T P})\end{array}$ & Sensitivity & Specificity & $\begin{array}{l}\text { Sensitivity } \\
\boldsymbol{p} \text { value }\end{array}$ & $\begin{array}{l}\text { Specificity } \\
\boldsymbol{p} \text { value }\end{array}$ \\
\hline Carlie & 56 & 1 & 1 & 47 & 7 & 87.5 & 97.9 & $7.25 \mathrm{E}-06$ & Not defined \\
Isabella & 56 & 4 & 5 & 43 & 4 & 50 & 89.6 & $1.47 \mathrm{E}-02$ & Not defined \\
Jake & 56 & 1 & 1 & 47 & 7 & 87.5 & 97.9 & $7.25 \mathrm{E}-06$ & Not defined \\
Juniper & 56 & 1 & 1 & 47 & 7 & 87.5 & 97.9 & $7.25 \mathrm{E}-06$ & Not defined \\
Nala & 56 & 1 & 1 & 47 & 7 & 87.5 & 97.9 & $7.25 \mathrm{E}-06$ & Not defined \\
Roscoe & 56 & 3 & 3 & 44 & 6 & 66.7 & 93.6 & 0.000155928 & Not defined \\
Total & & 11 & 12 & 275 & 38 & 77.6 & 95.8 & $1.98 \mathrm{E}-23$ & Not defined \\
\hline
\end{tabular}

Assumes binomial distribution prob success $=0.14$ \#Trials $=8$ per dog

$D A D s$ diabetes alert dogs, $F N$ false negative, $F P$ false positive, $T N$ true negative, $T P$ true positive

study, the dogs had a short break after four trials, allowing clearance of their olfactory senses (5) in our current study, we used The Pet Tutor which allowed the dogs to be enclosed in a room without a person, thus minimizing human interaction/distraction. The trial reported in our previous abstract took place with handlers in the same room as the dog.

Despite our current trial setup, we acknowledge that there are limitations to our study. The use of the Pet Tutor and placing the dogs inside a closed room for the study minimized cues from the trainer; however, we cannot completely assure no clues could have been sensed by the dog. Another problem is our use of a small number of dogs. Larger studies are needed. Finally, anytime an animal is used for study, there may be differences in performance due to inherent differences between animals studied, and those in a normal daily environment. Not all dogs will perform as well as these. Our study dogs received consistent instruction from a highly skilled trainer. DADs placed in a home environment need to have consistent reinforcement from their owners to remain accurate. Thus, careful selection of the patient and ongoing patient instruction are necessary for success.

Our study reinforces previous reports [6-11] which document the ability of dogs to recognize Hypo. However, several reports have refuted the ability of service dogs [18], including DADs [19]. A recent study by Dehlinger et al. [20] reported that dogs were unable to recognize Hypo. Several problems with the study limit its usefulness. In Dehlinger's trial, three DADs which had been previously trained and placed with T1D patients were removed from their homes for an in vitro study in which they were asked to alert to Hypo samples from three T1D patients "unfamiliar" to the DAD. Thus, the DADs were placed in an unfamiliar setting and given Hypo samples not belonging to their owner, despite their ongoing work with one person. Although trainers routinely begin training with multiple Hypo samples, once the DAD is ready to be placed with a specific patient, the training samples only come from the future owner. This ensures that the DAD works for his/her person and helps ensure the best service for the particular owner. It is well known that dogs can be affected by changes in 
their environment, by differences in training commands or voice tenor, or by their own fatigue. Use of both unfamiliar samples and an unfamiliar environment significantly limits the usefulness of this trial in predicts the ability of DADs. Of additional concern is the lack of documentation regarding the ongoing training and reinforcement of skill for the DADs.

There are reports of both success and failure of DADs, and there have been reports of "fake" service dogs of all types. Clearly, there is a need for standardized assessment of DADs. The Penn-Vet group in the U.S. has recently had a press release stating that they will provide assessment. We strongly support this group's goal of accreditation and would like to see trainers pass specific certification examinations before advertising or selling to the public.

Dogs have been well recognized for ability to help humans due to their keen sense of smell. The most familiar forms of scent training are the hunting dog and dogs used to sniff out illicit drugs. Dogs are also used to sniff out explosives and other contraband. Recent attention has been given to dogs as assistance in the medical field. Dogs have already been used for detection of cancer $[17,21]$ and detection of seizures. Some other medical uses include mobility assist and guiding blind people. Publications have also cited the indirect benefit of dogs in lowering blood pressure and providing stress relief; as well as encouraging exercise [22]. Each of these is pertinent benefits for people with diabetes. Standardization of DAD training and continued study of their accuracy are needed to further this important field.

\section{CONCLUSION}

Our current study supports evidence that dogs appear to have full capability to recognize and alert to Hypo samples from patients with T1D.
More studies are needed to elucidate best practices for training DADs, and ultimately to identify the chemical signature that the dogs detect.

\section{ACKNOWLEDGMENTS}

There was no sponsorship provided for the study. Article processing charges were paid for by Eli Lilly.

Authorship: All named authors meet the International Committee of Medical Journal Editors (ICMJE) criteria for authorship for this manuscript, take responsibility for the integrity of the work as a whole, and have given final approval to the version to be published.

Personnel acknowledgement: The authors thank Karla Villenes for administrative support during manuscript submission.

Disclosures. Dana S. Hardin declares that she works for the Eli Lilly and Company, but has no conflict of interest.

Author Wes Anderson works for Eli Lilly and Company and holds the patent for Pet Tutor ${ }^{\mathrm{TM}}$. Author Jennifer Cattet declares that she is the owner and head trainer for Medical Mutts.

Compliance with ethics guidelines. We have received approval of the institutional review board of St. Vincent Hospital, Indianapolis Indiana for collection techniques used to obtain Hypo samples from patients with diabetes. All institutional (Medical Mutts) and published guidelines (Assistance Dogs International) for use of positive reinforcement for the care and use of the dogs were followed.

Open Access. This article is distributed under the terms of the Creative Commons Attribution-NonCommercial 4.0 International License (http://creativecommons.org/licenses/ 
by-nc/4.0/), which permits any noncommercial use, distribution, and reproduction in any medium, provided you give appropriate credit to the original author(s) and the source, provide a link to the Creative Commons license, and indicate if changes were made.

\section{REFERENCES}

1. Briscoe VJ, Davis SN. Hypoglycemia in type 1 and type 2 diabetes: physiology, pathophysiology, and management. Clin Diabetes. 2006;24(3):115-21.

2. Tu E, Twigg SM, Semsarian C. Sudden death in type 1 diabetes: the mystery of the 'dead in bed' syndrome. Int J Cardiol. 2010;138(1):91-3 Epub 2008 Aug 3.

3. Wild D, et al. A critical review of the literature on fear of hypoglycemia in diabetes: implications for diabetes management and patient education. Patient Educ Couns. 2007;68:10-5.

4. Doll W, Pieber T, Farret A, Place J, Renard E, Bruttomesso D, Filippi A, Avogaro A, Arnolds S, Benesch C, Heinemann L, DeVries JH. AP@home consortium. Accuracy and reliability of continuous glucose monitoring systems: a head-to-head comparison. Diabetes Technol Ther. 2013;15(8): 722-7.

5. Juvenile Diabetes Research Continuous Glucose Monitoring Study Group. Effective glucose monitoring in a clinical care environment, evidence from the Juvenile Diabetes Research Foundation Continuous Glucose Monitoring (JDRF-CGM) Trial. Diabetes Care. 2010;33(1):17-22.

6. Wells DL, Lawson SW, Siriwardena AN. Canine responses to hypoglycemia in patients with type 1 diabetes. J Altern Complement Med. 2008;14(10): 1235-41.

7. Hardin DS, Hillman D, Cattet J. Hypoglycemia alert dogs-innovative assistance for people with type 1 diabetes. Diabetes. 2012;61(1):A99-100. doi:10. 2337/db12-378-655.

8. Rooney NJ, Morant S, Guest C. Investigation into the Value of Trained Glycaemia Alert Dogs to Clients with Type I Diabetes. PLoS One. 2013;8(8):e69921.

9. Gonder-Frederick L, Rice P, Warren D, Vajda K, Shepard J. Diabetic Alert Dogs: a preliminary survey of current users. Diabetes Care. 2013;36(4):e47.
10. Chen $M$, et al. Non-invasive detection of hypoglycaemia using a novel, fully biocompatible and patient friendly alarm system. BMJ. 2000;321(7276):1565-8.

11. Hardin DS, Cattet J, Anderson W, Skrivanek Z. Can diabetes alert dogs truly detect hypoglycemia? Diabetes. 2013;262(1):A-2967. doi:10.2337/db13388-679.

12. http://www.appliedanimalbehaviour.com/article/ S0168-1591(07)00395-4/abstract. Last Accessed Sep 5, 2015.

13. http://www.behaviorworks.org/files/articles/What's\% 20Wrong\%20with\%20this\%20Picture.pdf. Last Accessed Sep 5, 2015.

14. American Diabetes Association Workgroup on Hypoglycemia. Defining and reporting hypoglycemia in diabetes: a report from the American Diabetes Association workgroup on hypoglycemia. Diabetes Care. 2005;28(5):1245-9.

15. http://www.appliedanimalbehaviour.com/article/ S0168-1591(07)00395-4/abstract. Last Accessed Sep 5, 2015.

16. Horowitz A, editor. Domestic Dog Cognition and Behavior. Simon Gadbois and Catherine Reeve. Canine Olfaction: Scent, Sign, and Situation. Springer-Verlag, Berlin, Heidelberg; 2014.

17. McCulloch M, Jezierski T, Broffman M, Hubbard A, Turner K, Janecki T. Diagnostic accuracy of canine scent detection in early- and late-stage lung and breast cancers. Integr Cancer Ther. 2006;5(1):30-9.

18. National Public Radio(2014) Four-legged impostors give service dog owners pause. http://www.npr.org/ 2013/09/11/221017467/four-legged-impostors-giveservice-dog-owners-pauseHealthCentral. Accessed 30 Sept 2015.

19. Diabetes Alert Dogs Don't Detect Hypoglycemic Scent (2013) http://www.healthcentral.com/ diabetes/c/110/162286/diabetes-detect-hypoglycemia. Last Accessed Sep 5, 2015.

20. Dehlinger $\mathrm{K}$, et al. Can trained dogs detect a hypoglycemic scent in patients with type 1 diabetes? Diabetes Care. 2013;36:e98.

21. Willis CM, Britton LE, Harris R, Wallace J, Guest $\mathrm{CM}$. Volatile organic compounds as biomarkers of bladder cancer: sensitivity and specificity using trained sniffer dogs. Cancer Biomark. 2011;8(3): $145-53$.

22. Barker SB, Wolen AR. The benefits of human-companion animal interaction: a review. J Vet Med Educ. 2008;35(4):487-95. 